\title{
EXTENSION OF A THEOREM OF GUDDER AND SCHELP TO POLYNOMIALS OF ORTHOMODULAR LATTICES
}

\author{
LADISLAV BERAN
}

Abstract. Consider a polynomial expression $p(b, c, \ldots, d)=e$ where any two of the elements $b, c, \ldots, d$ commute. If an element $a$ commutes with $e$, then $b$ commutes with $p(a, c, \ldots, d)$.

1. Introduction. In this paper, $\mathcal{L}=\left(L, \vee, \wedge,{ }^{\prime}, 0,1\right)$ always means an orthomodular lattice. If $a, b \in L$ and $a=(a \wedge b) \vee\left(a \wedge b^{\prime}\right)$, then $a, b$ are said to commute; in this case we write $a C b$.

First recall some useful properties of the relation $C$ (see e.g. [2, pp. 52-53]).

1.1. Lemma. (i) If $a, b, c \in L$ are such that $a C b$ and $a C c$, then $a C b \wedge c$ and $a C b \vee c$.

(ii) If $\{a, b, c\}=\{x, y, z\}$ is $a$ subset of $L$, and if $x C y$ and $x C z$, then $a \wedge(b \vee c)$ $=(a \wedge b) \vee(a \wedge c)$ and $a \vee(b \wedge c)=(a \vee b) \wedge(a \vee c)$.

A well-known result states that if $a, b \in L$ and $a C b$, then $b C a$. For orthomodular lattices a result of Gudder and Schelp [5, p. 235] can be simplified and formulated in the following way: If $a C b \wedge c$ and $b C c$, then $b C a \wedge c$. The author's paper arose as the result of attempts to generalize these two special situations.

2. Preliminaries. In what follows, $\left\{b, c_{2}, \ldots, c_{n}\right\}$ denotes a subset of $L$ where any two elements commute.

2.1. LEMMA. Let $p$ be an $n$-ary lattice polynomial. Then

(1) $p\left(b, c_{2}, \ldots, c_{n}\right)=B_{1}\left(b, c_{2}, \ldots, c_{n}\right) \wedge B_{2}\left(b, c_{2}, \ldots, c_{n}\right)$, where

(2) $B_{1}\left(y_{1}, x_{2}, \ldots, x_{n}\right)=\wedge\left(y_{1} \vee \bigvee\left(x_{i} \mid i \in I_{j}\right) \mid j \in J\right)$,

(3) $B_{2}\left(y_{1}, x_{2}, \ldots, x_{n}\right)=\wedge\left(\bigvee\left(x_{k} \mid k \in K_{t}\right) \mid t \in T\right)$

and where $I_{j}, J, K_{t}, T$ are finite sets.

Proof. By Lemma 1(ii), the sublattice generated by $b, c_{2}, \ldots, c_{n}$ is distributive and the assertion follows from [1, Theorem 12, p. 145].

2.2. Corollary. Let $e_{1}=d, e_{2}, \ldots, e_{n} \in L$ be such that $e_{i} C e_{j}$ for $1<i, j<n$ and suppose (1) is valid. Then

$$
p\left(d, e_{2}, \ldots, e_{n}\right)=B_{1}\left(d, e_{2}, \ldots, e_{n}\right) \wedge B_{2}\left(d, e_{2}, \ldots, e_{n}\right)
$$

where $B_{1}$ and $B_{2}$ are the same polynomials as in (2) and (3).

Received by the editors March 24, 1980.

AMS (MOS) subject classifications (1970). Primary 06A30.

Key words and phrases. Orthomodular lattice, lattice polynomial.

(c) 1981 American Mathematical Society 0002-9939/81/0000-0153/\$01.75 
2.3. Proposition. If $a, b \in L$ are such that $a C b \wedge c$ and $b C c$, then $b C a \wedge c$.

Proof. Since $a=[a \wedge(b \wedge c)] \vee\left[a \wedge(b \wedge c)^{\prime}\right]$, we have

$$
a^{\prime}=\left(a^{\prime} \vee b^{\prime} \vee c^{\prime}\right) \wedge\left[a^{\prime} \vee(b \wedge c)\right]
$$

Thus

$$
a^{\prime} \vee c^{\prime}=\left\{\left(a^{\prime} \vee b^{\prime} \vee c^{\prime}\right) \wedge\left[a^{\prime} \vee(b \wedge c)\right]\right\} \vee c^{\prime}
$$

However, $a^{\prime} \vee b^{\prime} \vee c^{\prime}>a \wedge\left(b^{\prime} \vee c^{\prime}\right)=\left[a^{\prime} \vee(b \wedge c)\right]^{\prime}$ and it follows $a^{\prime} \vee(b \wedge$ c) $C a^{\prime} \vee b^{\prime} \vee c^{\prime}$. Similarly, $c^{\prime} C a^{\prime} \vee b^{\prime} \vee c^{\prime}$. By Lemma 1.1(ii),

$$
a^{\prime} \vee c^{\prime}=\left(a^{\prime} \vee b^{\prime} \vee c^{\prime}\right) \wedge\left[a^{\prime} \vee c^{\prime} \vee(b \wedge c)\right]
$$

But $c^{\prime} \vee(b \wedge c)=c^{\prime} \vee b$, by Lemma 1.1(ii). Therefore

$$
a^{\prime} \vee c^{\prime}=\left(a^{\prime} \vee c^{\prime} \vee b^{\prime}\right) \wedge\left(a^{\prime} \vee c^{\prime} \vee b\right)
$$

and, finally, $a \wedge c=[(a \wedge c) \wedge b] \vee\left[(a \wedge c) \wedge b^{\prime}\right]$.

3. Key lemma and main theorem. We begin this section with the following technical lemma.

3.1. Lemma. Let $p$ be an n-ary lattice polynomial and let (1) be valid. Then for every $a \in L$

$$
D_{1} \wedge D_{2}<p\left(a, c_{2}, \ldots, c_{n}\right)<D_{2}
$$

where

$$
\begin{aligned}
& D_{1}=\wedge\left(\bigvee\left(c_{i} \mid i \in I_{j}\right) \mid j \in J\right), \\
& D_{2}=\wedge\left(\bigvee\left(c_{k} \mid k \in K_{t}\right) \mid t \in T\right)
\end{aligned}
$$

Proof. Since $p$ is a lattice polynomial, by [3, Lemma 6, p. 33] we have

$$
p\left(0, c_{2}, \ldots, c_{n}\right)<p\left(a, c_{2}, \ldots, c_{n}\right)<p\left(1, c_{2}, \ldots, c_{n}\right) .
$$

By Corollary 2.2,

$$
p\left(0, c_{2}, \ldots, c_{n}\right)=B_{1}\left(0, c_{2}, \ldots, c_{n}\right) \wedge B_{2}\left(0, c_{2}, \ldots, c_{n}\right)=D_{1} \wedge D_{2} .
$$

Moreover,

$$
p\left(1, c_{2}, \ldots, c_{n}\right)=B_{1}\left(1, c_{2}, \ldots, c_{n}\right) \wedge B_{2}\left(1, c_{2}, \ldots, c_{n}\right)=D_{2} .
$$

3.2. THEOREM. Let $c_{1}, c_{2}, \ldots, c_{n}$ and $a$ be elements of an orthomodular lattice. If $c_{i} C c_{j}$ for any $1<i, j<n$ and if $a C p\left(c_{1}, c_{2}, \ldots, c_{n}\right)$ where $p$ is an $n$-ary lattice polynomial, then

$$
c_{k} C p\left(c_{1}, c_{2}, \ldots, c_{k-1}, a, c_{k+1}, \ldots, c_{n}\right)
$$

for every $k=1,2, \ldots, n$.

Proof. We may assume that $k=1$. Let $b=c_{1}$. By assumption, $a C p\left(b, c_{2}, \ldots, c_{n}\right)$. From Lemma 1.1(i), $c_{i} C p\left(b, c_{2}, \ldots, c_{n}\right)$ for every $i=2, \ldots, n$. By the same lemma we also have $p\left(a, c_{2}, \ldots, c_{n}\right) C p\left(b, c_{2}, \ldots, c_{n}\right)$. That is,

$$
B_{1}\left(b, c_{2}, \ldots, c_{n}\right) \wedge B_{2}\left(b, c_{2}, \ldots, c_{n}\right) C p\left(a, c_{2}, \ldots, c_{n}\right) \text {. }
$$

Since $b C c_{i}$ for every $i=2, \ldots, n$,

$$
B_{1}\left(b, c_{2}, \ldots, c_{n}\right)=b \vee\left(\wedge\left(\bigvee\left(c_{i} \mid i \in I_{j}\right) \mid j \in J\right)\right)=b \bigvee D_{1}
$$


Replacing $B_{2}\left(b, c_{2}, \ldots, c_{n}\right)$ by $D_{2}$ we see that

$$
\left(b \vee D_{1}\right) \wedge D_{2} C p\left(a, c_{2}, \ldots, c_{n}\right) \text {. }
$$

By Lemma 1.1(ii), $b C D_{1}$ and $b C D_{2}$. Hence $\left(b \wedge D_{2}\right) \vee\left(D_{1} \wedge D_{2}\right) C p\left(a, c_{2}, \ldots, c_{n}\right)$ and also $b \wedge D_{2} C D_{1} \wedge D_{2}$. In view of the assertion dual to Proposition 2.3, $b \wedge D_{2} C p\left(a, c_{2}, \ldots, c_{n}\right) \vee\left(D_{1} \wedge D_{2}\right)$. Then, by Lemma 3.1, $b \wedge$ $D_{2} C p\left(a, c_{2}, \ldots, c_{n}\right)$. Similarly, $b C p\left(a, c_{2}, \ldots, c_{n}\right) \wedge D_{2}=p\left(a, c_{2}, \ldots, c_{n}\right)$.

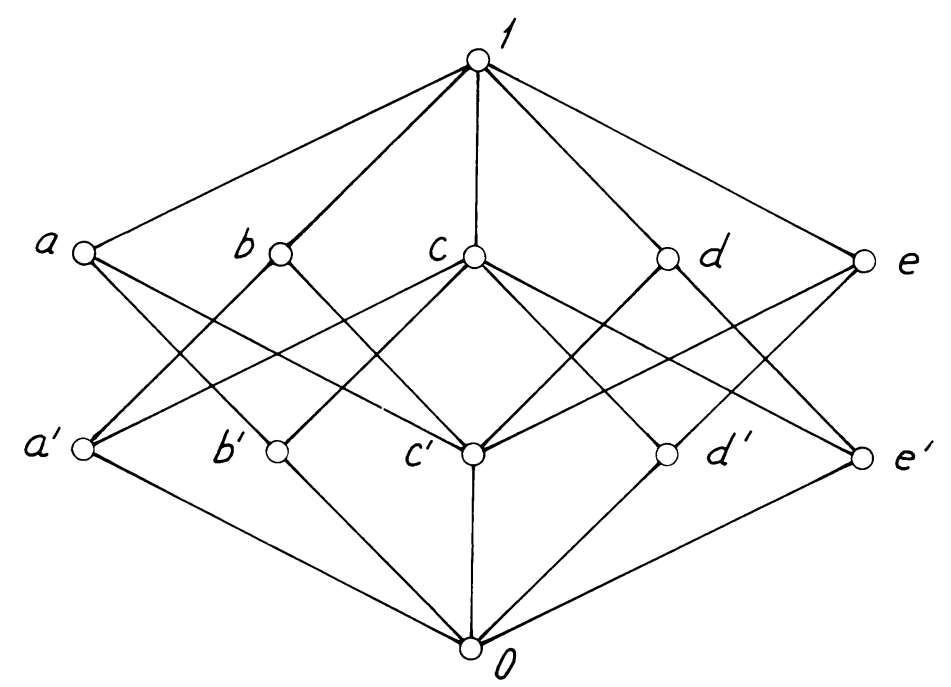

FIgURE 1

REMARK. We conclude by observing that the assertion of Theorem 3.2 is optimal in the following sense: If $q$ is a polynomial in $\vee, \wedge$ and ', then an analogue of Theorem 3.2 need not be valid. This can easily be seen by considering the orthomodular lattice constructed by Greechie's classical method [4] from two copies of $2^{3}$ (cf. Figure 1). Let $q(x, y)=(x \wedge y) \vee x^{\prime}$. Here we have $a C q\left(d^{\prime}, e\right)=$ 1 but $d^{\prime}$ and $q(a, e)=b$ do not commute.

\section{REFERENCES}

1. G. Birkhoff, Lattice theory, 2nd ed., Amer. Math. Soc. Colloq. Publ., Amer. Math. Soc., Providence, R.I., 1948. MR 10, p. 673.

2. __ Lattice theory, 3rd ed., Amer. Math. Soc. Colloq. Publ., vol. 25, Amer. Math. Soc., Providence, R.I., 1967. MR 37 \#2638.

3. G. Grätzer, Lattice theory: First concepts and distributive lattices, Freeman, San Francisco, Calif., 1971. MR 48 \#184.

4. R. J. Greechie, On the structure of orthomodular lattices satisfying the chain condition, J. Combin. Theory 4 (1968), 210-218. MR 37 \#2641.

5. S. P. Gudder and R. H. Schelp, Coordinatization of orthocomplemented and orthomodular pasets, Proc. Amer. Math. Soc. 25 (1970), 229-237. MR 41 \#3336.

Department of Algebra, Charles University, Sokolovskí 83, 18600 Prague 8, CzbchoSLOVAKIA 